\title{
The effect of acute back muscle fatigue on postural control strategy in people with and without recurrent low back pain
}

\author{
Ege Johanson • Simon Brumagne • Lotte Janssens • \\ Madelon Pijnenburg • Kurt Claeys • Mati Pääsuke
}

Received: 22 December 2009/Revised: 28 March 2011/Accepted: 16 April 2011/Published online: 1 May 2011

(C) Springer-Verlag 2011

\begin{abstract}
Back muscle fatigue decreases the postural stability during quiet standing, but it is not known whether this fatigue-induced postural instability is due to an altered proprioceptive postural control strategy. Therefore, the aim of the study was to evaluate if acute back muscle fatigue may be a mechanism to induce or sustain a suboptimal proprioceptive postural control strategy in people with and without recurrent low back pain (LBP). Postural sway was evaluated on a force platform in 16 healthy subjects and 16 individuals with recurrent LBP during a control (Condition 1) and a back muscle fatigue condition (Condition 2). Back muscle fatigue was induced by performing a modified Biering-Sørensen test. Ankle and back muscle vibration, a potent stimulus for muscle spindles, was used to differentiate proprioceptive postural control strategies during standing on a stable and unstable support surface, where the latter was achieved by placing a foam pad under the feet. Ankle signals were predominantly used for postural control in all subjects although, in each condition, their influence was greater in people with LBP compared to healthy subjects $(p<0.001)$. The latter group adapted their postural control strategy when standing on an unstable surface so that input from back muscles increased $(p<0.001)$. However, such adaptation was not observed
\end{abstract}

\footnotetext{
E. Johanson · M. Pääsuke

Faculty of Exercise and Sport Sciences, Institute of Exercise Biology and Physiotherapy, University of Tartu, 5 Jakobi Street, 51014 Tartu, Estonia

S. Brumagne $(\square) \cdot$ L. Janssens $\cdot$ M. Pijnenburg $\cdot$ K. Claeys Musculoskeletal Research Unit, Faculty of Kinesiology and Rehabilitation Sciences, Department of Rehabilitation Sciences, University of Leuven (K.U.Leuven),

Tervuursevest 101, 3001 Leuven, Belgium

e-mail: simon.brumagne@faber.kuleuven.be
}

when the back muscles were fatigued. Furthermore, people with LBP continued to rely strongly on ankle proprioception regardless of the testing conditions. In conclusion, these findings suggest that impaired back muscle function, as a result of acute muscle fatigue or pain, may lead to an inability to adapt postural control strategies to the prevailing conditions.

Keywords Postural strategy - Proprioception .

Muscle vibration $\cdot$ Sensory reweighting $\cdot$ Postural balance

\section{Introduction}

Optimal postural control is essential to perform daily activities. The central nervous system (CNS) must identify and selectively focus on the sensory inputs (visual, vestibular, proprioceptive) that provide the functionally most reliable signals [1]. Muscle fatigue may decrease the reliability of the proprioceptive signals [2] and therefore the CNS might down-weight this sensory input and/or up-weight proprioceptive signals of other muscles to control posture [3, 4].

People with recurrent low back pain (LBP) have been observed to have motor control impairments [5] and altered lumbosacral proprioceptive acuity $[6,7]$, which might be a causative factor in their postural instability. Healthy individuals normally maintain postural stability using a "multisegmental" control strategy $[1,4,8]$. In contrast, people with LBP seem to use a more rigid postural strategy (i.e., ankle-steered strategy) to control postural balance resulting in postural instability when postural demands increase $[9,10]$. The underlying mechanisms of this motor control impairment and postural instability are not completely clear yet. 
Postural control might be negatively influenced by muscle fatigue. Muscle fatigue can be defined as a decreased force-generating capacity [11] and may be caused by peripheral changes or by a failure of the CNS to drive the motoneurons adequately [10]. It possibly influences postural control due to altered muscle contractile efficiency [11, 12], proprioceptive acuity [2] and cortical control $[13,14]$. Excessive fatigability of the back extensor muscles is common among people with chronic LBP [1517]. The fatigue-related changes in muscle stiffness may reduce the capacity of the paraspinal muscles to stabilize the spine [18]. Furthermore, Taimela et al. concluded that lumbar muscle fatigue impaired lumbar position sense in patients with LBP and healthy subjects [19]. Some studies showed that lumbar extensors fatigue resulted in an increased postural sway in healthy individuals [20, 21]. However, to the authors' knowledge, no studies have examined the possible relationship between back muscle fatigue and the selection of a proprioceptive postural control strategy in healthy individuals and in people with recurrent LBP.

Therefore, the aim of the present study was to evaluate whether acute back muscle fatigue alters the proprioceptive postural control strategy in people with and without recurrent LBP when standing on a stable or unstable support surface [10, 22]. Muscle vibration, known as a strong stimulus of muscle spindles [23, 24], was used to differentiate proprioceptive postural control strategies [10]. The modified Biering-Sørensen test was used to induce acute fatigue of back muscles $[15,17]$. We hypothesized that acute back muscle fatigue in healthy individuals induces a shift to a similar postural control strategy as in people with recurrent LBP. This may be the result of a similar underlying mechanism.

\section{Materials and methods}

\section{Subjects}

A total of 32 individuals voluntarily participated in this study including 16 people ( 11 women, 5 men) with a history of LBP and 16 control subjects ( 11 women, 5 men). Their ages ranged from 18 to 33 years. A medical screening by a physician was performed to include and exclude subjects. Study participants were excluded if they had LBP with a non-musculoskeletal etiology, musculoskeletal injuries of the lower limbs, previous spinal surgery, a history of neurological disease, specific balance or coordination problems, a history of cerebral trauma or if they were using any pain relieving medication. Individuals were included in the LBP group if they had experienced non-specific mechanical LBP for more than 6 months, reported at least $6 / 100$ on the
Oswestry Disability Index, Version 2 (ODI) [25] and had at least three self-reported recurrent episodes of LBP. None was undergoing regular medical treatment or physical therapy for their LBP at the time of testing or in the last 6 months. The patients with LBP were recruited from the Department of Physical Medicine and Rehabilitation, University Hospitals of Leuven, where they had sought medical attention for LBP. Individuals were included in the control group if they had no history of LBP and an ODI equal to $0 / 100$. These healthy subjects were recruited from a student population. All subjects gave their informed consent. A physical activity questionnaire was administered. Participants were asked to rate their pain on a numerical rating scale (NRS) anchored with "no pain at all" and "unbearable pain" $(0-10)$.

The characteristics of subjects are presented in Table 1. All procedures were approved by the institutional medical research ethical committee and were applied with respect to the Declaration of Helsinki (Ethical Principles for Medical Research Involving Human Subjects).

\section{Movement analysis}

Postural sway characteristics were measured using a sixchannel force plate (Bertec, OH, USA). Force plate data were sampled at $500 \mathrm{~Hz}$ using a Micro 1401 data acquisition system and Spike2 software (Cambridge Electronic Design, UK) and low pass filtered with a cutoff frequency of $5 \mathrm{~Hz}$.

\section{Muscle vibration}

Muscle vibration, as a strong stimulation for muscle spindles [23, 24], was used to appraise the role of proprioception in postural control. During vibration, an illusion of

Table 1 Characteristics of people with recurrent LBP and healthy subjects

\begin{tabular}{llcl}
\hline & $\begin{array}{l}\text { Control group } \\
(n=16)\end{array}$ & $\begin{array}{l}\text { LBP group } \\
(n=16)\end{array}$ & $p$ value \\
\hline Age (years) & $22.7 \pm 1.7$ & $22.0 \pm 1.1$ & NS \\
Height (cm) & $174.7 \pm 9.6$ & $172 \pm 10.7$ & NS \\
Weight (kg) & $66.8 \pm 12.5$ & $65.5 \pm 9.6$ & NS \\
PAI (Baecke) & $8.8 \pm 2.5$ & $9.4 \pm 1.2$ & NS \\
Duration of LBP (years) & 0 & $3.7 \pm 2.2$ & \\
NRS pain (before) & 0 & $1.6 \pm 1.8$ & \\
NRS pain (after) & 0 & $5.2 \pm 2.5$ & \\
ODI-2 & 0 & $14.4 \pm 6.3$ & \\
\hline
\end{tabular}

$L B P$ low back pain; $P A I$ physical activity index questionnaire $(0-15)$; $N R S$ pain numerical rating scale for pain before and after the experiment (0-10); ODI-2 Oswestry Disability Index version $2(0-100)$ at the moment of testing. $N S$ not significant $(p>0.05)$; data are presented as mean $\pm \mathrm{SD}$ 


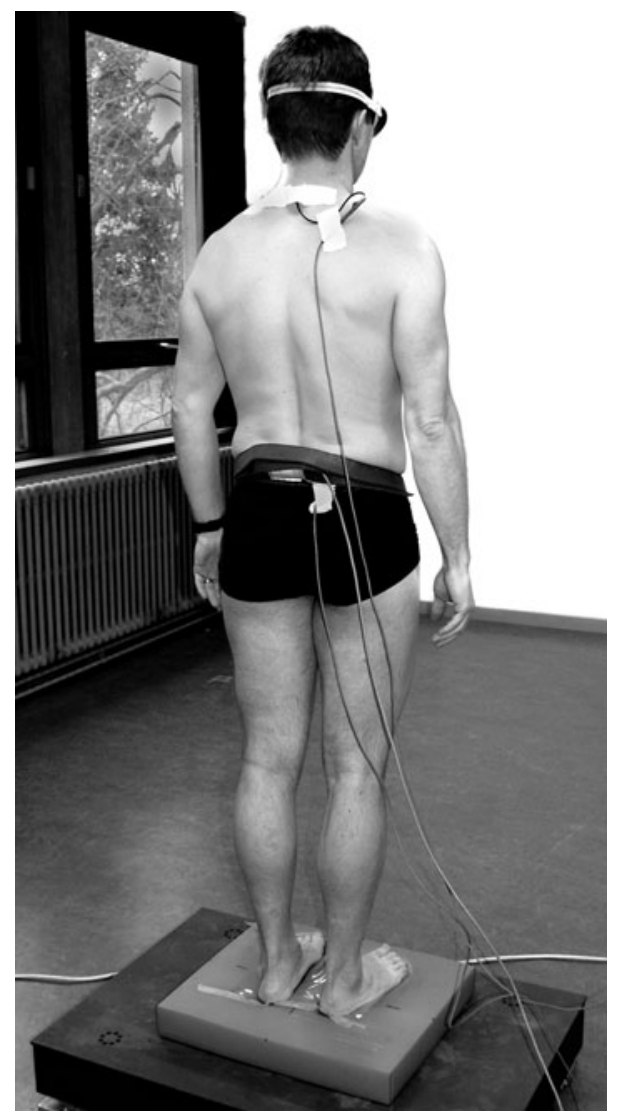

Fig. 1 Experimental setup. Lumbar multifidus vibration during upright standing on an unstable support surface ("foam")

muscle lengthening alters the proprioceptive sense $[24,26]$. During standing in healthy individuals, vibration of triceps surae (TS) muscles induces an involuntary body sway in backward direction, whereas lumbar multifidus (MF) muscle vibration produces a forward body sway [4, 10]. Displacement of the center of pressure $(\mathrm{CoP})$ specifies how the subject makes use of proprioceptive signals from the vibrated muscle to control posture. Therefore, two muscle vibrators (Maxon motors, Switzerland) were used. Vibration was applied bilaterally to TS muscles (at the Achilles tendon-muscle belly transition) or MF muscle (at the segmental level L5-S1), respectively (Fig. 1). The frequency of vibration was $60 \mathrm{~Hz}$ and the amplitude was approximately $0.5 \mathrm{~mm}$. Trials lasted $60 \mathrm{~s}$. Muscle vibration started $15 \mathrm{~s}$ after the start of trial and lasted for $15 \mathrm{~s}$.

\section{Test procedure}

Two test conditions were used to evaluate postural control: (1) control condition (Day 1) and (2) back muscle fatigue condition (Day 2). Each condition involved six trials (Table 2). All trials were performed on stable and unstable support surfaces. For the unstable support surface trials, a "foam" (Airex balance pad) was used to decrease the
Table 2 Experimental protocol

1. Control (day 1)

1.A. Upright stance-stable support surface

1.A.1. Without vision

1.A.2. Without vision, bilateral triceps surae muscle vibration

1.A.3. Without vision, bilateral lumbar multifidus muscle vibration

1.B. Upright stance-unstable support surface (foam)

1.B.1. Without vision

1.B.2. Without vision, bilateral triceps surae muscle vibration

1.B.3. Without vision, bilateral lumbar multifidus muscle vibration

2. Back muscle fatigue (day 2)

2.A. Upright stance-stable support surface

2.A.1. Without vision

2.A.2. Without vision, bilateral triceps surae muscle vibration

2.A.3. Without vision, bilateral lumbar multifidus muscle vibration

2.B. Upright stance-unstable support surface (foam)

2.B.1. Without vision

2.B.2. Without vision, bilateral triceps surae muscle vibration

2.B.3. Without vision, bilateral lumbar multifidus muscle vibration

reliability of ankle proprioception, so the CNS should rely on other proprioceptive signals for postural control such as from the back muscles $[10,22]$. Back muscle fatigue was used to evaluate if this may be a mechanism to induce changes in proprioceptive postural strategies. During the trials, the subject had to stand barefoot on a force plate (or on "foam" on the force plate) with the arms loosely hanging along the body (Fig. 1). The heels were $10 \mathrm{~cm}$ apart with the forefeet in a free splayed out position. Vision was occluded by non-transparent glasses and subjects were instructed to remain immobile, but relaxed.

In the muscle vibration trials, data were collected for $15 \mathrm{~s}$ prior to the start of vibration; muscle vibration lasted for $15 \mathrm{~s}$ and data collection continued for $30 \mathrm{~s}$ after cessation of vibration.

In the back muscle fatigue condition, a modified Biering-Sørensen back endurance test $[15,17]$ was performed and immediately followed by the same six postural control trials used in the first (control) condition (Fig. 2). To insure that the force platform measurements were obtained in a genuine fatigued state, the fatiguing test took place beside the force platform. During the modified Biering-Sørensen test, the subjects lay in a prone position on a treatment bench, with their feet, knees and hips strapped to the bench. The belts were tightened as firmly as possible while considering the subject's level of comfort. The upper body hanged over the bench, with the hands crossed over the chest. 


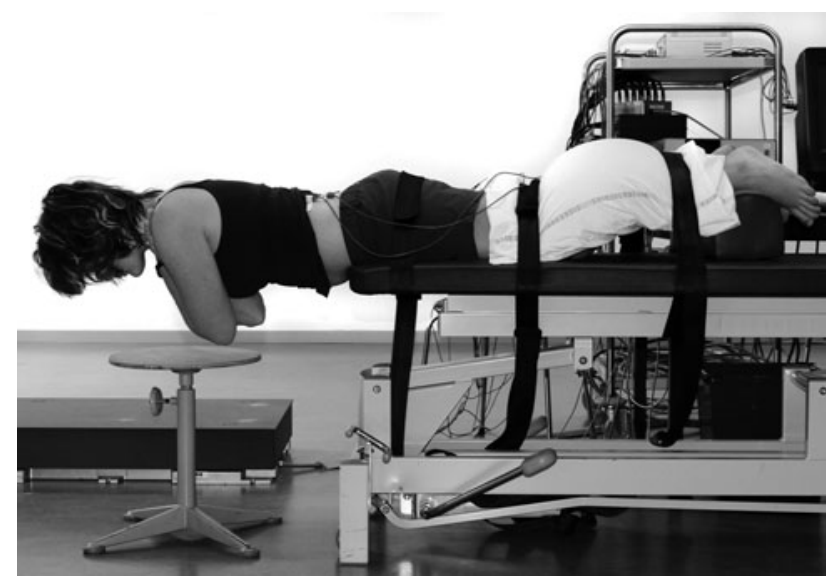

Fig. 2 The back endurance test

The instructed task was to lift the upper body in the horizontal plane with the head and neck in neutral position. The subjects were asked to maintain this position until exhaustion. The test was ended when the subjects could no longer maintain their upper body in the horizontal plane despite verbal encouragement. Endurance time was recorded in seconds. Participants were asked to rate their back pain on an NRS (0-10) and their perceived effort on an adapted Borg scale (0-10).

While the back endurance test was performed, surface electromyography (EMG) was recorded from the iliocostalis lumborum pars thoracis (IL) and MF muscles (Myosystem, USA). The pairs of surface electrodes (Medicotest blue sensor, INC, USA) were placed $2 \mathrm{~cm}$ apart, over the muscle belly, and following the direction of the muscle fibers. To reduce cross-talk signals from adjacent muscles, the electrode positions of the IL and MF muscles were at the intersection of the line corresponding to the muscle fiber orientation and horizontal lines through the spinous process of L2 (IL) and L5 (MF), respectively. A ground electrode was placed over the right malleolus lateralis. The EMG data were amplified $(1,000 \times)$, band-pass filtered $(10-500 \mathrm{~Hz})$ and sampled at $2,000 \mathrm{~Hz}$ using a Micro1401 data acquisition system and Spike2 software (Cambridge Electronic Design, UK).

\section{Data reduction and statistical analysis}

Postural sway characteristics were recorded from the force plate readings using Spike2 and Microsoft Excel software. Positions of the COP in the anterior-posterior direction were calculated from the raw force plate data using the equation: $\mathrm{COP}=\mathrm{Mx} / \mathrm{Fz}$. $\mathrm{Mx}$ is the moment in the sagittal plane and $\mathrm{Fz}$ is the vertical force (i.e., weight of the subject). Further data reduction was performed by calculating the root mean square (RMS) values of the COP positions, as a measure of postural stability, and the mean values, to appraise the directional effect of muscle vibration on COP position. The COP positions in the muscle vibration trials were analyzed over two epochs: the $15 \mathrm{~s}$ preceding (baseline) and the $15 \mathrm{~s}$ during muscle vibration. Deduction of the mean COP position during vibration from the mean $\mathrm{COP}$ position during baseline provides the effect of muscle vibration on postural control. Positive values correspond to forward COP displacement and negative values with backward COP displacement. In addition, ratios of the COP displacement during the TS muscle vibration trials versus MF muscle vibration trial were calculated to determine the proprioceptive postural control strategy using the equation: RW TS/MF $=$ absolute $\mathrm{TS} /($ abs $\mathrm{TS}+$ abs MF). Where $\mathrm{RW}$ is the relative proprioceptive weighting, abs TS is the absolute value of mean COP displacement during TS muscles vibration and abs MF is the absolute value of mean COP displacement during MF vibration. A score of zero means $100 \%$ reliance on lumbar muscles proprioception in postural control. In contrast, a score of one means $100 \%$ reliance on proprioception of the ankle muscles in postural control [10]. Based on the results obtained in our previous studies [4, 10], a sample size of 13 subjects per group was determined to provide adequate power ( 0.80 with a twotailed alpha level of 0.05 ) to detect a clinically relevant difference in the center of pressure displacement (mean $=0.022 \pm 0.024 \mathrm{~m}$ ).

To determine back muscle fatigue, EMG mean power frequency (MPF) was calculated [27]. Differences between the two groups, conditions and trials were analyzed using a repeated measures analysis of variance (ANOVA/MANOVA). Post hoc analysis (Tukey) was performed on significant main and interaction effects to calculate specific effects. The level of statistical significance was set at $p<0.05$. The statistical analysis was performed with Statistica 9.0 (Statsoft, OK, USA).

\section{Results}

\section{Control condition}

The unstable support surface caused RMS values to increase significantly, compared to the stable support surface, in individuals with recurrent LBP $(p<0.0001)$ but not in the control subjects $(p>0.05)$.

When standing on the unstable support surface, people with recurrent LBP showed significantly larger posterior sways than controls $(p<0.001)$ when ankle muscle vibration was applied, but significantly smaller anterior sways compared to controls $(p<0.0002)$ when back muscle vibration was used (Table 3; Fig. 3).

Based on the proprioceptive weighting ratios, people with LBP showed a significantly more ankle-steered 
Table 3 Center of pressure displacements of people with LBP and healthy subjects

\begin{tabular}{|c|c|c|c|c|}
\hline Condition & $\begin{array}{l}\text { LBP group } \\
(n=16) \\
\text { Mean } \pm \text { SD (m) }\end{array}$ & $\begin{array}{l}\text { Control group } \\
(n=16) \\
\text { Mean } \pm \text { SD }(\mathrm{m})\end{array}$ & $F$ value & $p$ value \\
\hline \multicolumn{5}{|l|}{ Control } \\
\hline PS (rms) & $0.079 \pm 0.039$ & $0.071 \pm 0.040$ & 1.67 & NS \\
\hline TS (mean) & $-0.117 \pm 0.051$ & $-0.093 \pm 0.015$ & 3.79 & NS \\
\hline MF (mean) & $0.014 \pm 0.016$ & $0.036 \pm 0.022$ & 3.47 & 0.01 \\
\hline PS F (rms) & $0.123 \pm 0.033$ & $0.081 \pm 0.025$ & 6.64 & 0.02 \\
\hline TS F (mean) & $-0.103 \pm 0.036$ & $-0.056 \pm 0.019$ & 8.86 & 0.001 \\
\hline MF F (mean) & $0.015 \pm 0.010$ & $0.054 \pm 0.022$ & 4.24 & 0.0002 \\
\hline \multicolumn{5}{|l|}{ Fatigued } \\
\hline PS (rms) & $0.077 \pm 0.038$ & $0.082 \pm 0.024$ & 1.67 & NS \\
\hline TS (mean) & $-0.100 \pm 0.035$ & $-0.100 \pm 0.015$ & 3.79 & NS \\
\hline MF (mean) & $0.014 \pm 0.006$ & $0.029 \pm 0.018$ & 3.47 & NS \\
\hline PS F (rms) & $0.136 \pm 0.038$ & $0.131 \pm 0.037$ & 6.64 & NS \\
\hline TS F (mean) & $-0.106 \pm 0.026$ & $-0.096 \pm 0.021$ & 8.86 & NS \\
\hline MF F (mean) & $0.015 \pm 0.008$ & $0.039 \pm 0.015$ & 4.24 & 0.003 \\
\hline
\end{tabular}

$F$ values represent the interaction effects and $p$ values the Tukey post hoc tests

$S D$ standard deviation; $P S$ postural stability trial; $r m s$ root mean square; TS triceps surae muscles vibration; $M F$ lumbar multifidus muscle vibration; $F$ while standing on a "foam" support; $L B P$ low back pain; $N S$ not significant

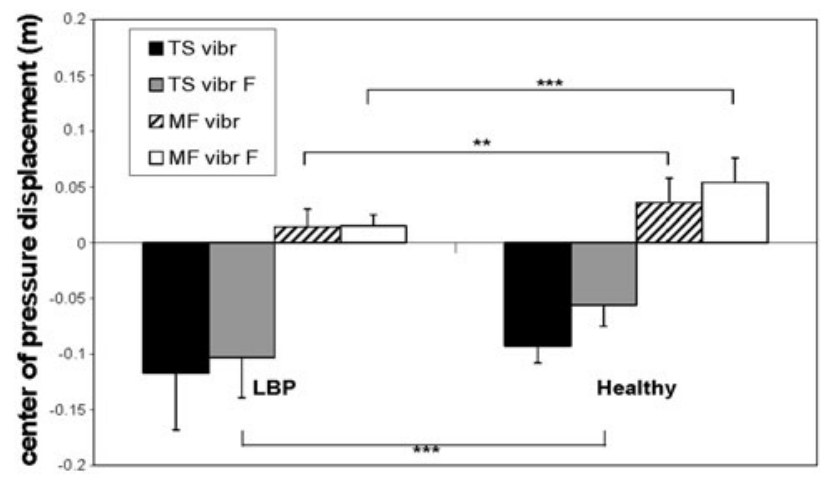

Fig. 3 Means and standard deviations of the anterior-posterior sways for the muscle vibration trials in Condition 1. Note that the negative center of pressure displacements indicate posterior sway and positive displacements indicate anterior sway. TS vibr, triceps surae muscles vibration; TS vibr F, triceps surae muscles vibration while standing on a "foam" support; MF vibr, lumbar multifidus muscles vibration; MF vibr $\mathrm{F}$, lumbar multifidus muscles vibration while standing on a "foam" support; LBP, low back pain; ** $p<0.01$; *** $p<0.001$ proprioceptive control strategy compared to healthy subjects (Table 4).

Back muscle fatigue condition

\section{Back muscle fatigability}

People with recurrent LBP had significantly shorter endurance time during the Biering-Sørensen test compared to healthy subjects $(127.4 \pm 36.7$ and $184.0 \pm 40.4 \mathrm{~s}$, respectively) $(p<0.01)$. The MPF of the back muscles in both groups declined significantly during the back endurance test $(p<0.05)$ (Fig. 4). There was no significant difference in perceived effort (Borg scores) after this test between the LBP and healthy groups $(9.2 \pm 1.2$ and $8.3 \pm 1.3$, respectively) $(F(1.30)=3.44, p>0.05)$.

People with LBP showed a significant increase in pain after the test $(F(1.15)=5.076, p<0.05)$, but healthy

Table 4 Relative proprioceptive weighting ratios of people with LBP and healthy subjects

\begin{tabular}{llllr}
\hline Condition & & $\begin{array}{l}\text { LBP group }(n=16) \\
\text { Mean } \pm \text { SD }(\%)\end{array}$ & $\begin{array}{l}\text { Control group }(n=16) \\
\text { Mean } \pm \text { SD }(\%)\end{array}$ & $F$ value \\
\hline Control & RW TS/MF & $0.85 \pm 0.07$ & $0.73 \pm 0.11$ & 12.31 \\
& RW TS/MF F & $0.86 \pm 0.07$ & $0.52 \pm 0.16$ & 58.69 \\
Fatigued & RW TS/MF & $0.86 \pm 0.09$ & $0.78 \pm 0.11$ & 0.11 \\
& RW TS/MF F & $0.86 \pm 0.09$ & $0.72 \pm 0.10$ & 0.000 \\
& & & 0.007 & 0.000 \\
\hline
\end{tabular}

$S D$ standard deviation; $R W$ relative weighting; $T S$ triceps surae muscles vibration; $M F$ lumbar multifidus muscle vibration; $F$ while standing on a "foam" support; $L B P$ low back pain 


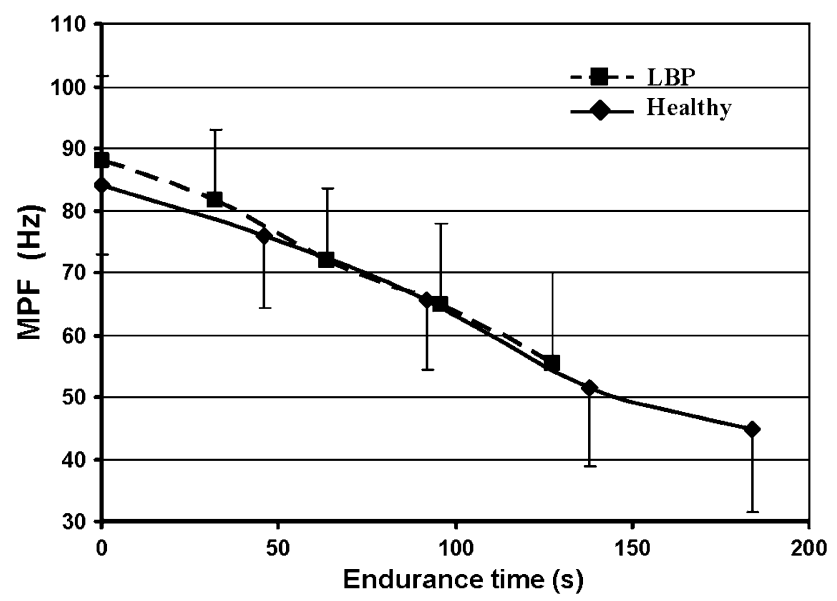

Fig. 4 Means and standard deviations of the changes with time in mean power frequency of the lumbar multifidus muscle in healthy subjects and people with LBP during the back muscle endurance test

controls had no pain after back endurance test $(p>0.05)$ (Table 1).

\section{Postural stability and proprioceptive control strategies after back muscle fatigue}

Back muscle fatigue induced a significant decrease in postural stability in healthy subjects when standing on an unstable support surface compared to the control condition (mean RMS values: $0.131 \pm 0.037 \mathrm{~m}$ vs. $0.081 \pm 0.025 \mathrm{~m}$, respectively; $p<0.05$ ). People with LBP maintained their decreased postural stability after back muscle fatigue was induced (mean RMS values: $0.136 \pm 0.038 \mathrm{~m}$ (on foam) and $0.123 \pm 0.033 \mathrm{~m} ; p>0.05$ ).

Back muscle fatigue had no significant influence on proprioceptive control during both the TS muscle vibration and MF muscle vibration trials in healthy subjects and people with LBP while standing on a stable support surface $(p>0.05)$. However, when standing on an unstable support surface, acute back muscle fatigue induced an increased backward sway during ankle muscle vibration in healthy subjects compared to the control condition $(p<0.001)$. Moreover, back muscle fatigue induced a significant decrease in anterior sway during MF vibration in the healthy controls compared to the control condition when standing on the "foam" support $(0.039 \pm 0.015 \mathrm{~m}$ vs. $0.054 \pm 0.022 \mathrm{~m}$, respectively; $p<0.05$ ). Despite this decrease, people with LBP still showed significantly smaller forward sways during MF vibration compared to the healthy individuals $(p<0.01)$ (Fig. 5).

The proprioceptive weighting ratios showed that when healthy subjects were standing on an unstable support surface, and their back muscles were fatigued, they relied significantly more on ankle proprioception for postural

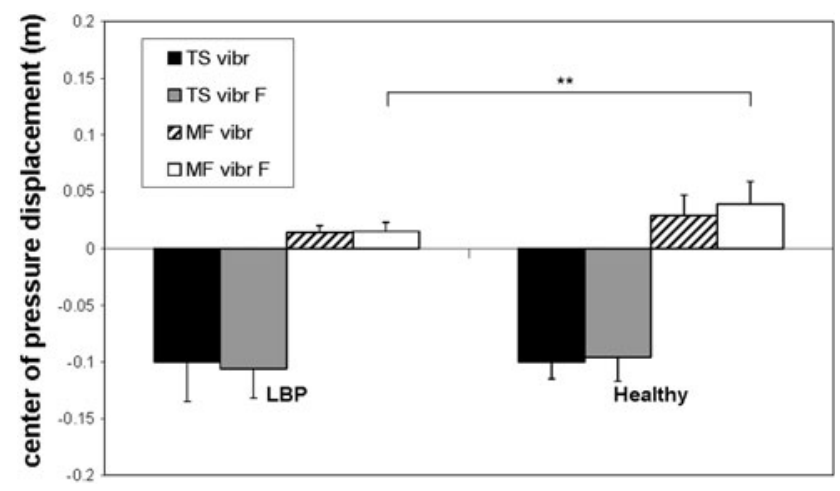

Fig. 5 Means and standard deviations of the anterior-posterior sways for the muscle vibration trials in Condition 2. TS vibr, triceps surae muscles vibration; TS vibr $\mathrm{F}$, triceps surae muscles vibration while standing on a "foam" support; MF vibr, lumbar multifidus muscles vibration; MF vibr $\mathrm{F}$, lumbar multifidus muscles vibration while standing on a "foam" support; LBP, low back pain; ** $p<0.01$

control than they did when their back muscles were not fatigued $(p<0.001)$ (Table 4$)$.

In contrast, back muscle fatigue did not have a significant additional influence on relative proprioceptive weighting ratios in people with LBP compared to healthy controls when standing on an unstable support surface $(p>0.05)$.

\section{Discussion}

Acute back muscle fatigue may be a mechanism to induce changes in proprioceptive postural strategy. The main result of this study is that healthy individuals after back muscle fatigue were significantly more dependent on ankle proprioception while standing on an unstable support surface in comparison with the control condition. This suggests that in healthy subjects, back muscle fatigue induced a shift to a more ankle-steered proprioceptive postural control strategy when standing on an unstable support surface, as used by people with recurrent LBP.

\section{Control condition}

People with LBP were more dependent on ankle signals in comparison to healthy subjects during the control condition. An explanation for this reliance could be reduced lumbosacral proprioception [6, 7]. This probably leads to a refocusing of proprioceptive sensitivity from the trunk to the ankles [4, 10]. Another possible but not mutually exclusive explanation is increased antagonistic cocontraction of the trunk muscles to stabilize the spine [18], which might lead to a reduced multi-segmental control strategy [9]. 
Moreover, our results demonstrated that people with recurrent LBP sustained their reliance on ankle proprioception for controlling posture while standing on an unstable support surface. An unstable support surface decreases the acuity of ankle signals [22]. Therefore, their sustained reliance on ankle proprioception showed their inability to switch to a more appropriate proprioceptive postural control strategy, as demonstrated by other studies $[9,10]$, leading to decreased postural stability. In contrast, based on the lower proprioceptive weighting ratios, healthy controls seemed to make more use of other proprioceptive signals, in addition to those from the ankles, which is more in line with the multi-segmental control model $[1,8]$. These results confirmed our previous findings [4, 10].

\section{Back muscle fatigue condition}

In the present study, healthy individuals had a longer endurance time of the back extensor muscles in comparison to people with recurrent LBP. These results are in agreement with previous studies [15-17]. Both the significant decline in MPF of the back muscles and the very high perceived effort scores after the back endurance test in both groups suggested that real fatigue of the back muscles was induced.

However, despite the very high perceived effort scores, it is still important to take the possibility of a submaximal performance regarding back muscle endurance into consideration. Pain-related factors might contribute to the perception of a maximal effort in people with LBP [28]. Moreover, despite the significantly shorter endurance time in patients, the rate of decline in mean MPF was similar in the two groups suggesting that the patients may not have reached the same level of fatigue as the healthy individuals. Most people with LBP reported significantly more pain after the back muscle fatigue test, so we could not exclude that they stopped earlier due to this increase in pain.

Back muscle fatigue in healthy individuals resulted in a significantly stronger reliance on proprioceptive signals from the ankles for controlling posture during quiet standing on "foam", resulting in a decreased postural stability. In comparison with the control condition, significantly larger posterior sways have been shown during TS muscle vibration while standing on an unstable support surface. An increased sway due to lumbar muscle fatigue has already been shown by some studies [20, 21]. This can be explained by the negative influence of fatigue on the muscle receptors and thereby on proprioception [2, 14]. Due to lumbar muscle fatigue, proprioceptive acuity can decrease, which leads to inaccurate signals about lumbar spine position and movement [19]. Under simple (nonfatigued) postural conditions, greater dependence upon proprioceptive input from the ankles is the norm, and increased input from back muscle spindles only becomes important when the stance is unstable, in which case healthy controls adapt their strategy accordingly but people with LBP do not. It is the ability to adapt postural control strategy in unstable conditions, which then appears to be lost in the healthy subjects when their back muscle is fatigued.

\section{Clinical implications}

The results of this study suggested that back muscle fatigue may cause a proprioceptive reweighting from the trunk to the ankles when standing on an unstable support surface. Thus, reducing the fatigability of the back muscles might reverse this effect. The possibility to rely on both ankle and trunk proprioceptive signals (i.e., a multi-segmental control strategy) to control posture may lead to a greater flexibility in adapting to different postural circumstances [1, 29]. In contrast, a dominant reliance on ankle signals implies a lesser ability to adapt to more complex postural situations [9, 10]. Accordingly, a rigid postural control strategy may serve its purpose in restricting excessive spinal movement during simple, familiar postural tasks, but then becomes sub-optimal under more complex postural conditions when it actually induces larger spinal motions and excessive tissue loading [9].

\section{Limitations}

This study population may not be representative of a general patient population due to the young ages and low disability scores. Moreover, because of the young age, low disability and fairly moderate challenging postural tasks, these results may underestimate the postural control impairment that may be observed in a patient population of older age and with higher disability during the more demanding activities of daily life. Therefore, future studies with an older population and more disability must be conducted. We have some borderline significant results, which might be recognized as true differences by enlarging the sample. Spreading an experiment over 2 days is another possible limitation. A person's physical state might change during the time interval. Because of pragmatic reasons, the test order was not randomized. Despite the short rest periods between the trials, learning effects and general fatigue cannot be ruled out from affecting the results. In addition, back muscle fatigue might be recovered when performing postural control trials. However, the total duration of these trials is about $10 \mathrm{~min}$. So, complete recovery from back muscle fatigue cannot be expected in that time frame. Moreover, significant differences in postural strategy were observed when standing on the unstable support surface, which were the last trials. 


\section{Conclusions}

The results of this study showed that healthy individuals adopted a multi-segmental control strategy when postural instability was induced by using an unstable support surface. However, people with recurrent LBP continued to rely strongly on ankle proprioception resulting in decreased postural stability. Back muscle fatigue in healthy subjects impaired the ability to adapt their postural control strategy to the prevailing conditions and resulted in healthy individuals resorting to a similar postural strategy to that observed in patients with recurrent LBP when postural demands increased.

Acknowledgments This work was supported by grants from the Research Foundation-Flanders (FWO) (1.5.104.03 and G.0674.09). Ege Johanson is an international scholar of the Vlaamse Gemeenschap, Flanders, Belgium. Lotte Janssens is a doctoral fellow of FWO. Madelon Pijnenburg is a doctoral fellow of the Agency for Innovation by Science and Technology (IWT).

Conflict of interest None.

\section{References}

1. Allum JH, Bloem BR, Carpenter MG, Hulliger M, Hadders-Algra M (1998) Proprioceptive control of posture: review of new concepts. Gait Posture 8:214-242

2. Allen TJ, Proske U (2006) Effect of muscle fatigue on the sense of limb position and movement. Exp Brain Res 1:30-38

3. Carver S, Kiemel T, Jeka JJ (2006) Modeling the dynamics of sensory reweighting. Biol Cybern 95:123-134

4. Brumagne S, Cordo P, Verschueren S (2004) Proprioceptive weighting changes in persons with low back pain and elderly persons during upright standing. Neurosci Lett 366:63-66

5. Hodges PW, Richardson CA (1996) Inefficient muscular stabilization of the lumbar spine associated with low back pain. A motor control evaluation of transversus abdominis. Spine 21:2640-2650

6. Brumagne S, Cordo P, Lysens R, Verschueren S, Swinnen S (2000) The role of paraspinal muscle spindles in lumbosacral position sense in individuals with and without low back pain. Spine 25:989-994

7. Newcomer KL, Laskowski ER, Yu B, Johnson JC, An KN (2000) Differences in repositioning error among patients with low back pain compared with control subjects. Spine 25:2488-2493

8. Morasso PG, Schieppati M (1999) Can muscle stiffness alone stabilize upright standing? J Neurophysiol 82:1622-1626

9. Mok NW, Brauer SG, Hodges PW (2007) Failure to use movement in postural strategies leads to increased spinal displacement in low back pain. Spine 32:E537-E543
10. Brumagne S, Janssens L, Knapen S, Claeys K, Suuden-Johanson E (2008) Persons with recurrent low back pain exhibit a rigid postural control strategy. Eur Spine J 17:1177-1184

11. Bigland-Ritchie B, Johansson R, Lippold OC, Woods JJ (1983) Contractile speed and EMG changes during fatigue of sustained maximal voluntary contractions. J Neurophysiol 50:313-324

12. Duchateau J, Hainaut K (1985) Electrical and mechanical failures during sustained and intermittent contractions in humans. J Appl Physiol 58:942-947

13. Taylor JL, Butler JE, Allen GM, Gandevia SC (1996) Changes in motor cortical excitability during human muscle fatigue. J Physiol 15 490:519-28

14. Gandevia SC (2001) Spinal and supraspinal factors in human muscle fatigue. Physiol Rev 81:1725-1774

15. Biering-Sørensen F (1984) Physical measurements as risk indicators for low-back trouble over a 1-year period. Spine 9:106-119

16. Mannion AF, Connolly B, Wood K, Dolan P (1997) The use of surface EMG power spectral analysis in the evaluation of back muscle function. J Rehab Res Dev 34:427-439

17. Latimer J, Maher CG, Refshauge K, Colaco I (1999) The reliability and validity of the Biering-Sorensen test in asymptomatic subjects and subjects reporting current or previous nonspecific low back pain. Spine 24:2085-2089

18. Granata KP, Slota GP, Wilson SE (2004) Influence of fatigue in neuromuscular control of spinal stability. Hum Factors 46:81-91

19. Taimela S, Kankaanpaa M, Luoto S (1999) The effect of lumbar fatigue on the ability to sense a change in lumbosacral position. A controlled study. Spine 24:1322-1327

20. Vuillerme N, Anziani B, Rougier P (2007) Trunk extensor muscles fatigue affects undisturbed postural control in young healthy adults. Clin Biomech 22:489-494

21. Davidson BS, Madigan ML, Nussbaum MA (2004) Effects of lumbar extensor fatigue and fatigue rate on postural sway. Eur J Appl Physiol 93:183-189

22. Ivanenko YP, Solopova IA, Levik YS (2000) The direction of postural instability affects postural reactions to ankle muscle vibration in humans. Neurosci Lett 292:103-106

23. Roll JP, Vedel JP (1982) Kinaesthetic role of muscle afferents in man, studied by tendon vibration and microneurography. Exp Brain Res 47:177-190

24. Cordo PJ, Gurfinkel VS, Brumagne S et al (2005) Effect of slow, small movement on the vibration-evoked kinesthetic illusion. Exp Brain Res 167:324-334

25. Fairbank JCT, Pynsent PB (2000) The oswestry disability index. Spine 22:2940-2953

26. Goodwin GM, McCloskey DI, Matthews PB (1972) Proprioceptive illusions induced by muscle vibration: contribution by muscle spindles to perception? Science 175:1382-1384

27. Ng JK, Richardson CA (1996) Reliability of electromyographic power spectral analysis of back muscle endurance in healthy subjects. Arch Phys Med Rehabil 77:259-264

28. Tam GY, Yeung SS (2006) Perceived effort and low back pain in non-emergency ambulance workers: implications for rehabilitation. J Occup Rehabil 16:231-240

29. Schieppati M, Giordano A, Nardone A (2002) Variability in a dynamic postural task attests ample flexibility in balance control mechanisms. Exp Brain Res 29:E107-E112 